\title{
EVOLUCIÓN DEL CONCEPTO DE CAMBIO CLIMÁTICO Y SU IMPACTO EN LA SALUD PÚBLICA DEL PERÚ
}

\author{
Carlos A. Sánchez Zavaleta ${ }^{1, a}$
}

\begin{abstract}
RESUMEN
El término de "cambio climático" no es un concepto nuevo, pero su impacto en la salud pública está en continua revisión. Sabemos que el clima ya cambió y va seguir cambiando por los próximos siglos con el aumento de la temperatura global promedio, y el consecuente aumento del nivel del mar. Este hecho hace que los esfuerzos para la mitigación de la emisión de gases sean relevantes únicamente a muy largo plazo y para generaciones de humanos cuyos padres aun no nacen. Si hablamos de salud pública en el contexto de cambio climático, hablamos de adaptación. En el presente, los países más afectados por los efectos del cambio climático son justamente los países como el Perú, sin una huella ecológica significativa a nivel mundial, pero que son altamente sensibles a los efectos del clima. Sin proyecciones confiables del cambio en el clima, el impacto en la salud puede ser incierto y complicado. Sin embargo, a nivel local, cada distrito puede identificar sus propias vulnerabilidades y definir sus prioridades para asegurar la salud de su población. Existen y se pueden crear indicadores de salud ambiental para monitorizar qué tan bien nos estamos adaptando y qué tan preparados estamos para los cambios en el clima. La adaptación a los cambios del clima implica elevar las condiciones de vida, mejorar los sistemas de vigilancia epidemiológica y ampliar el acceso a servicios de salud. La lucha contra los efectos del cambio climático en salud pública es la lucha contra la pobreza y la desigualdad, y eso no es nada nuevo en el Perú.
\end{abstract}

Palabras clave: cambio climático, salud ambiental, Perú (fuente: DeCS BIREME)

\section{EVOLUTION OF THE CLIMATE CHANGE CONCEPT AND ITS IMPACT IN THE PUBLIC HEALTH OF PERU}

\begin{abstract}
The term "climate change" is not a new concept but its impact on public health is under constant review. We know that climate has already changed and will continue to change for centuries with the rise in average global temperature, and the associated rise in sea level. This fact makes mitigation efforts relevant only in the very long term and for generations of humans whose parents have not yet been born. When we talk about public health in the context of climate change, we are talking about adaptation. In the present, countries that are currently the most affected by climate change are precisely countries like Peru, without a significant carbon footprint at the global level but that are highly sensitive to the effects of climate. Without reliable climate projections, the health impact of climate change can be uncertain and complicated. Nevertheless, at the local level, every district can identify its vulnerabilities and define priorities to protect the health of its population. There are, and it can also be developed, environmental health indicators that can help monitor how well we are adapting and how prepared we are for changes in the climate. Adaptation to climate change implies improving living conditions, enhancing epidemiological surveillance systems and extending access to healthcare. The fight against the effects of climate change in public health is a fight against poverty and inequality, and that is nothing new in Peru.
\end{abstract}

Key words: climate change, environmental health, Peru (source: MeSH NLM)

\section{INTRODUCCIÓN}

El concepto de "cambio climático" no es un concepto nuevo. En 1992, la Convención Marco sobre Cambio Climático definió al "cambio climático" como: El cambio en el clima que es atribuible directa o indirectamente a la actividad humana que altera la composición de la atmosfera global y que se suma a la variabilidad natural del clima que se ha observado sobre períodos de tiempo comparables ${ }^{(1)}$. En 1999, el "calentamiento global" se definió como: El incremento gradual en las temperaturas promedio del aire cerca de la superficie y de los océanos desde mediados del siglo $X X$ y su continua proyección. Estas definiciones fueron, en general, mal recibidas porque incluían dos puntos controversiales: 1) el clima se estaba "calentando", y 2) los responsables podrían

Universidad Peruana de Ciencias Aplicadas. Lima, Perú.

a Licenciado en Biología, bachiller en Medicina. 
ser los seres humanos. Para muchos recién introducidos al tema, la existencia de nieve y el antecedente de eras glaciales eran incompatibles con tales afirmaciones $y$, en todo caso, el aumento de uno o dos grados de temperatura promedio no era un problema para personas con acceso a aire acondicionado. En efecto, el Primer Informe de Evaluación del Panel Intergubernamental para Cambio Climático (Intergovernmental Panel on Climate Change o IPCC por sus siglas en inglés) de 1990, había presentado pocas pruebas de observación respecto de una influencia climática antropógena perceptible. Desde entonces, la confianza en la evaluación de la contribución humana al reciente cambio climático ha ido en aumento. Seis años después, el Segundo Informe de Evaluación del IPCC concluyó que las pruebas sugerían una "influencia humana discernible" sobre el clima del siglo XX. El Tercer Informe de Evaluación en el año 2001 concluyó que: La mayor parte del calentamiento observado durante los últimos 50 años se debió, probablemente, al aumento en las concentraciones de gases de efecto invernadero ${ }^{(2)}$. Sin embargo, esto seguía sin convencer a las multitudes. La película "Una verdad incómoda" (An Inconvenient Truth) se estrena en el año 2006, creando controversia y reacciones mixtas. Mucha de la crítica entonces (e incluso ahora) era sobre cuánta seguridad había de que los culpables sean los seres humanos. Para separar controversias, en el año 2007, el cuarto informe del IPCC define el "cambio climático" como: cualquier cambio del clima en el tiempo, ya sea por variabilidad natural o como resultado de la actividad humana ${ }^{(3)}$. Es decir, obviando por un momento si es natural o artificial, el mensaje universal se vuelve priorizar el enfoque en el "cambio" del clima (no solo el calentamiento). Ese año, el IPCC y el exvicepresidente de los Estados Unidos Al Gore recibieron conjuntamente el premio Nobel de la Paz ${ }^{(4)}$. El debate sobre la responsabilidad humana persiste, pero cada vez menos personas cuestionan que el clima ha cambiado.

La realidad es que existe suficiente evidencia científica para justificar la noción de un calentamiento global. El efecto invernadero es un sistema natural que mantiene al planeta lo suficientemente caliente para permitir la vida. Se estima que sin el efecto invernadero la temperatura promedio del planeta sería de $-18{ }^{\circ} \mathrm{C}$. Los gases de efecto invernadero (GEI) naturales incluyen: vapor de agua, dióxido de carbono, metano, oxido nitroso y ozono, pero el dióxido de carbono $\left(\mathrm{CO}_{2}\right)$ es el gas antropogénico más importante de los GEl y tiene una vida media en la atmosfera de varios siglos.

Los niveles en los últimos 50 años se encuentran muy por encima de los niveles en cualquier otro período registrado en la historia del planeta. La Figura 1 muestra los niveles de $\mathrm{CO}_{2}$ en la atmósfera en los últimos 400 mil años antes del presente ${ }^{(5)}$. El IPCC propone que los niveles elevados de $\mathrm{CO}_{2}$ son responsables del cambio en el clima desde mediados del siglo XX. Previo a la Revolución Industrial, estos gases eran balanceados por procesos naturales (por ejemplo, el mar y las plantas absorben $\mathrm{CO}_{2}$ ), manteniendo su concentración en la atmósfera. Se estima que en los últimos 200 años, Norteamérica, Europa y la antigua Unión Soviética (cuya población actual conjunta representa el $20 \%$ de la población mundial) han contribuido al $80 \%$ de las emisiones de $\mathrm{CO}_{2}{ }^{\left({ }^{6}\right)}$. En tan solo un par de siglos, la humanidad ha conseguido perturbar indefinidamente el termostato natural del planeta. Aunque actualmente la mayoría de los esfuerzos a nivel mundial son para la "mitigación" de los GEI, ahora sabemos que este loable

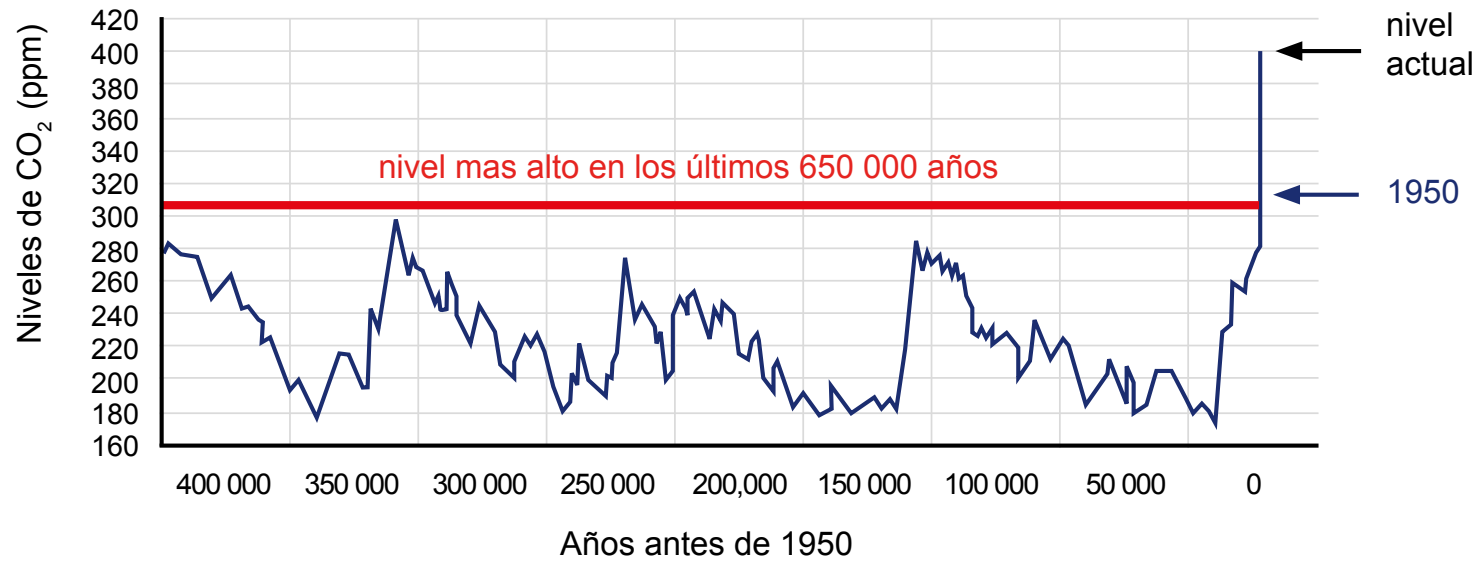

Traducido de: National Aeronautics and Space Administration (NASA).

Disponible en: http://climate.nasa.gov/evidence/

Figura 1. Cambios en dióxido de carbono en los últimos 400000 años 
objetivo es poco realista debido a la falta de voluntad de varios países. Los países que más han contaminado en el pasado (por ejemplo, Europa y Estados Unidos) se resisten a comprometer su presente para mantener su estilo de vida, y los nuevos países industriales (por ejemplo, China e India) se resisten a comprometer su futuro para seguir creciendo. Parece poco probable que se pongan de acuerdo. Mientras tanto, los países como el Perú, que no tienen una huella ecológica significativa a nivel mundial, pero que son altamente sensibles a los efectos del clima, son actualmente los más afectados ${ }^{(7)}$. El cambio climático no está en camino, el clima en el mundo ya cambió, va seguir cambiando, y hay que hacer algo al respecto.

Hace varios años que el IPCC ha estimado que el daño ya está hecho: existe un exceso de $\mathrm{CO}_{2}$ presente en la atmósfera que va demorar varios siglos en removerse y está asociado al aumento de la temperatura. Los modelos indican (Figura 2) que incluso en un escenario ideal si las emisiones industriales de $\mathrm{CO}_{2}$ llegaran a un pico (es decir, si logramos limitar la emisión mundial de GEI durante este siglo), el aumento de la temperatura, y el consecuente aumento del nivel del mar continuarán en ascenso por los próximos mil años. En la figura se puede ver que, en el caso imaginario donde los niveles de emisión de $\mathrm{CO}_{2}$ empezaran a descender en los próximos años (línea marrón), la estabilización de $\mathrm{CO}_{2}$ que ya ha sido emitido (línea púrpura), de la temperatura (línea roja), y de la expansión térmica del mar (línea verde), demorarán varios siglos ${ }^{\left({ }^{8}\right)}$. De acuerdo con las proyecciones del IPCC, los esfuerzos para la mitigación a nivel mundial son solo importantes a muy largo plazo, y solamente relevantes para generaciones de seres humanos cuyos padres todavía no han nacido. En términos de la salud pública de la población actual, la mitigación no es una opción costo-efectiva y, en ese contexto, el tema de mitigación se vuelve fútil. En lo que se refiere a la salud pública, es hora de adaptarse.

Ya en 1999 se anticipaba que para el año 2080 las concentraciones de $\mathrm{CO}_{2}$ en el aire se habrían duplicado (comparadas con las concentraciones en la era preindustrial), el mundo entero se calentaría en unos $3^{\circ} \mathrm{C}$ en promedio (aunque los continentes se calentarían mas que los océanos), y habrían cambios sustanciales en la precipitación, especialmente en los trópicos ${ }^{(9)}$. Esta tendencia a incrementar la temperatura promedio a nivel global no es lineal, y no quiere decir que cada año va ser subsecuentemente más caluroso que el anterior, hay variabilidad en estos datos. La Figura 3 presenta los promedios anuales de temperatura desde el año 1860 comparadas con el promedio de temperatura para los años 1961 a $1990{ }^{(10)}$. Se puede ver en la figura que los promedios anuales a veces se encuentran por encima del promedio y a veces por debajo del promedio, pero a partir de fines de los años 30 , existen más años con promedios anuales por encima del promedio 1961-1990. La tendencia de temperatura es hacia promedios mayores, y esa tendencia parece estar asociada a la curva ascendente de concentración de $\mathrm{CO}_{2}$. Las grandes variaciones de un año al otro se pueden explicar con fenómenos climáticos naturales como El Niño, pero la tendencia general se explica primordialmente por la contaminación antropogénica de $\mathrm{CO}_{2}$. El "cambio" va a ser diferente para cada región y la adaptación al cambio climático va a ser también un esfuerzo regional. Por ejemplo, países como Bangladesh y Vietnam podrían perder hasta $20 \%$ de su territorio bajo el agua, ${ }^{(11,12)}$ mientras que otros países como Siria y Jordania tendrán cada vez menos agua ${ }^{(13)}$. Un país tan megadiverso como el Perú tendrá un poco de todo: sequía e inundaciones, olas de calor y friajes, aumento del nivel del mar y pérdida de nevados.

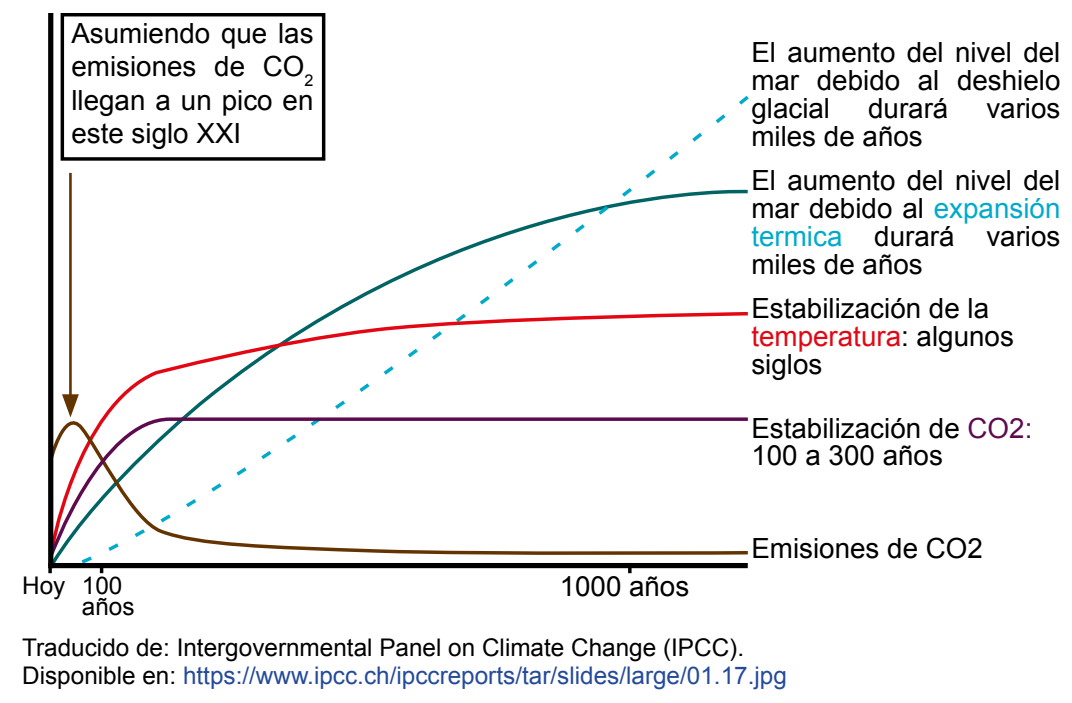

Figura 2. Temperatura global y concentración de dióxido de carbono 


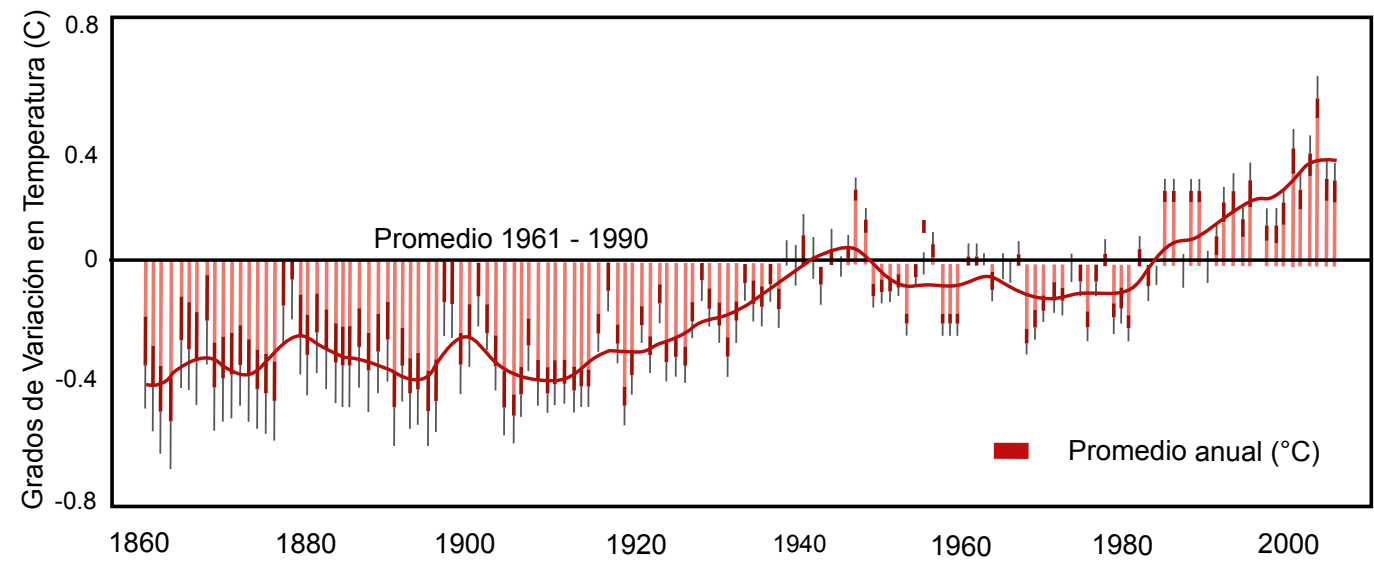

Traducido de: Intergovernmental Panel on Climate Change (IPCC).

Disponible en: http://www.mongabay.com/images/2006/graphs/ipcc-temp.jpg

Figura 3. Temperatura global y concentración de dióxido de carbono

\section{IMPACTO EN LA SALUD}

La Convención Marco de las Naciones Unidas sobre Cambio Climático (CMNUCC), desarrollada en Nueva York en 1992, establece un marco general para los esfuerzos internacionales encaminados a abordar el problema del cambio climático. El Perú se adhiere a la CMNUCC en el año 1992, y se suscribe al Protocolo de Kioto ${ }^{(14)}$ en 1998: con el primero reconoce que el cambio climático es una realidad y con el segundo establece que como país firmante reportará periódicamente los avances en la implementación local de los objetivos de dicha convención. El objetivo supremo de la CMNUCC es estabilizar las concentraciones de GEI en la atmósfera a un nivel que impida que el clima se perjudique. Sin embargo, pocos recuerdan que también es uno de los "compromisos" de la CMNUCC: Cooperar en los preparativos para la adaptación a los impactos del cambio climático... con miras a reducir al mínimo los efectos adversos en la economía, la salud pública y la calidad del medioambiente (1). La CMNUCC, desde su concepción, propone evaluar el impacto en la salud pública, y el proceso de adaptación a los efectos del cambio climático, pero el tema del impacto en la salud hasta ahora no ha sido priorizado. El cuarto reporte del IPCC en el año 2007 identificaba los siguientes como las principales vías en las que el cambio climático podría afectar la salud de las personas: 1) desnutrición por la reducción en la producción de alimentos; 2) lesiones y enfermedades por una mayor intensidad en las olas de calor e incendios, y 3) cambios en la temporalidad y distribución espacial de las enfermedades infecciosas ${ }^{(3)}$. Se sabe ahora que el tema es más complicado. El quinto (último) reporte del IPCC en el año 2014, amplia el tema del compromiso de la salud, además de otras consecuencias como la pérdida de capacidad laboral y productividad laboral reducida debido al aumento de temperaturas ${ }^{(7)}$.

A corto plazo, el aumento de temperatura en las próximas décadas, implica que los días más calientes excederán las temperaturas actuales por un amplio margen y habrá un incremento en el número de personas que viven en condiciones que son tan extremas que la habilidad del cuerpo humano para mantener su temperatura durante la actividad física se verá comprometida (al menos durante una parte del año) y el trabajo en exteriores sin protección simplemente no será posible. El sobrecalentamiento del cuerpo humano no es una consecuencia exclusiva del aumento de temperatura. El aumento en la humedad relativa aumenta también el riesgo de enfermedad por calor, pues evita la evaporación del sudor, impidiendo que el cuerpo se enfríe. Esta es la razón por la cual algunas definiciones de "ola de calor" incluyen la medición de la humedad relativa adicionalmente al incremento de temperatura pues implica un mayor riesgo para la salud. Tampoco es efecto de un solo día caluroso, son varios días a lo largo de los cuales el cuerpo humano va perdiendo su homeostasis.

Luego de la ola de calor que azotó Europa en agosto de 2003, se determinó que la población con mayor riesgo de mortalidad en París eran las personas ancianas con enfermedades crónicas que vivían solas en pobres condiciones socioeconómicas ${ }^{(15)}$. Los niños y los ancianos están en mayor riesgo, pero hay tiempo para la prevención. Ese estudio determinó que simples medidas de prevención antes del evento podrían salvar más vidas que una masiva respuesta de los hospitales durante el evento. Lo mismo se había concluido durante la ola de calor en Chicago casi una década antes ${ }^{(16)}$. Por 
otro lado, el cambio climático también incluye cambios en la precipitación, por ejemplo: nuevas zonas en sequía; por lo que la idea de zonas "demasiado calientes para la vida humana" se hace escalofriantemente posible. Es necesario recordar también que no solo la población expuesta ocupacionalmente (agricultores, mineros, constructores, etc.) está en un mayor riesgo, las personas que viven en ciudades también podrían estar en riesgo aumentado debido al fenómeno de "isla urbana de calor" que puede aumentar la temperatura ambiental en unos $2-4{ }^{\circ} \mathrm{C}$ (en promedio) adicionales al aumento de temperatura debido al cambio climático.

La Organización Mundial de la Salud (OMS) reconoce que el cambio climático influye en los determinantes sociales y medioambientales de la salud como: el aire limpio, agua potable, alimentos suficientes y una vivienda segura ${ }^{(17)}$. Se estimaba que para el año 2004 el calentamiento mundial que se ha producido desde los años setenta habría causado un exceso de más de 140000 defunciones anuales y que muchas de las enfermedades más mortíferas, como las diarreas, la malnutrición, la malaria y el dengue, son muy sensibles al clima, y era de prever que se agravarán con el cambio climático. Es razonable suponer que las regiones con malas infraestructuras sanitarias serán las menos capacitadas para prepararse 0 adaptarse a estos cambios. En su último informe, el IPCC ha concluido que "en el futuro" los países más afectados por los efectos del cambio climático son justamente los que "actualmente" se ven más afectados por el clima. Ese es el caso del Perú.

El impacto del cambio climático sobre la salud de las personas se vuelve un tema mas complejo cuando se toma en cuenta que la "salud" no es solo la ausencia de enfermedad sino es el bienestar general de la persona y que el "ambiente" que rodea a cada persona es el principal condicionante de ese bienestar. Por ejemplo, los problemas económicos producidos por el cambio climático en países en desarrollo perpetuarán la pobreza y las malas condiciones de vida, que son importantes factores condicionantes de la salud de una población. Otros riesgos que se asocian a los escenarios climáticos menos optimistas incluyen la producción de alimentos y el acceso a agua potable ${ }^{(18)}$. Del mismo modo, los problemas con desastres naturales repercutirán en el acceso de las personas a una vivienda adecuada (que protege de ciertas enfermedades); mientras los problemas en la agricultura y la pesca se traducirán en problemas en la nutrición de las personas (que afecta el desarrollo y la inmunidad). Aquellas poblaciones (por ejemplo, comunidades indígenas) que dependan más de estas actividades (la agricultura y la pesca) se verán directamente afectadas. Es importante para el Perú reconocer cuales son sus poblaciones más vulnerables a estos efectos en la salud.
En los Estados Unidos, los impactos en la salud reportados el 2014 en la Evaluación Nacional del Clima (National Climate Assessment o NCA por sus siglas en inglés) ${ }^{(7)}$ incluyen:

- Contaminación del aire (mayor cantidad de ozono a nivel de la superficie terrestre y mayor cantidad de material particulado).

- Aeroalérgenos (veranos más largos y mayores concentraciones de $\mathrm{CO}^{2}$ equivale a mayor producción de polen por algunas plantas).

- Incendios forestales (periodos de sequías mas largos causarán incendios mayores con el consiguiente mayor producción de humo y la disminución de la calidad del aire).

- Extremos de temperatura (olas de calor o inviernos menos severos).

- Extremos de precipitación (lluvia abundante o sequías).

- Enfermedades transmitidas por vectores (la enfermedad de Lyme, fiebre por dengue, virus del oeste del Nilo, fiebre moteada de las montañas Rocallosas, peste, y tularemia son enfermedades que ya existen en los Estados Unidos).

- Enfermedades diarreicas transmitidas por agua y alimentos (salmonelosis, criptosporidium).

- Seguridad alimentaria (menor producción de alimentos, aumento de los costos, y menor valor nutricional).

- Salud mental (desórdenes asociados al estrés).

Es evidente que todos estos impactos no se van a dar a lo largo de la extensión completa del territorio de los Estados Unidos. Algunas áreas se verán más afectadas que otras, y de manera diferente. A ese nivel local, algunas poblaciones $\mathrm{o}$ comunidades vulnerables se verán en mayor riesgo. Por ejemplo, en el NCA se hace una mención especial a las minorías indígenas (por ejemplo, los esquimales de Alaska), no solo porque se les considera en su mayoría de un nivel socioeconómico bajo, sino porque, además, su estilo de vida ya se está viendo afectado por los cambios en el clima.

El calentamiento global podría tener algunos efectos beneficiosos localizados, como una menor morbilidad/ mortalidad en invierno en las regiones templadas y un aumento de la producción de alimentos en determinadas zonas ${ }^{(7,20)}$. Sin embargo, se considera que el mayor impacto del cambio climático a mediano plazo (de aquí al año 2050) en la salud de las personas será la exacerbación de enfermedades existentes, y el mayor riesgo se 
encuentra en las poblaciones cuya salud y bienestar son afectadas actualmente por el clima ${ }^{(7)}$. La Tabla 1 enumera una clasificación inicial donde se puede ver que los efectos en la salud son diversos y se solapan ${ }^{(19)}$. Por ejemplo, los eventos climáticos severos (tormentas y huracanes) pueden afectar directamente la salud causando lesiones y muerte,

Tabla 1. Clasificación del Impacto en la salud humana del cambio climático, NIH 2010

\footnotetext{
Mortalidad y morbilidad asociada a calor

Incremento en frecuencia y severidad de olas de calor (deshidratación, golpe de calor, shock de calor)

Mortalidad y morbilidad asociada al clima

Huracanes, inundaciones, sequías, e incendios (lesiones, heridas, disrupción de servicios básicos)

Enfermedad transmitida por agua

Contaminación del agua (químicos y patógenos)

Enfermedad transmitida por vectores y zoonosis

Expansión de la distribución del vector (malaria, dengue)

Acortamiento de los periodos de incubación del patógeno (Fasciola)

Reubicación de grandes poblaciones humanas

Enfermedad transmitida por alimentos y nutrición

Desnutrición por escasez de los alimentos

Contaminación de la comida (alimentos marinos: químicos, biotoxinas y microbios, cosechas con pesticidas)

Efectos en el desarrollo humano

Desnutrición: reducción de alimentos en agricultura y pesca Exposición a contaminantes tóxicos y biotoxinas (uso incrementado de pesticidas en alimentos, aumento en brotes de algas peligrosas)

Enfermedad neurológica

Riesgo incrementado por:

Biotoxinas neurotóxicas (por los brotes de algas peligrosas)

Metales (de áreas industriales inundadas)

Pesticidas (utilizados en agricultura en respuesta a cambios)

Enfermedad mental y desordenes asociados al stress

Desplazamiento de poblaciones enteras

Daños a la propiedad

Pérdidas de seres queridos

Estrés crónico

\section{Enfermedad cardiovascular e infarto}

Exacerban la enfermedad cardiovascular existente:

- Estrés por calor

- Carga corporal de partículas en el aire

- Cambio en distribución de vectores de enfermedades

Asma, alergias y enfermedades respiratorias

Aumento en la exposición humana al: polen, moho, toxinas marinas, contaminación del aire, polvo

Cáncer

Incremento en duración e intensidad a radiación ultravioleta Vías de exposición para químicos y toxinas

Traducido y resumido de: National Institute of Health (NIH). A Human Health Perspective on climate change. 2010
}

pero también pueden afectar a corto plazo el suministro de agua causando enfermedades diarreicas y neurológicas por contaminación de agua y alimentos; pueden aumentar el riesgo de enfermedades transmitidas por vectores $\mathrm{y}$, finalmente, a largo plazo pueden causar problemas en el desarrollo, enfermedades mentales, y cáncer. De la misma manera, la desnutrición puede ser causada por la disrupción de los servicios de abastecimiento, por la baja producción en la agricultura o por el bajo contenido proteico de los alimentos (todos son impactos del cambio climático).

La Tabla 2 muestra una clasificación mas reciente del IPCC para los efectos del cambio climático en la salud de las personas ${ }^{(7)}$. Los impactos se pueden dividir en tres grandes grupos: los impactos mediados por el ecosistema (aquellos que surgen de un desequilibrio en las relaciones entre varios factores además del clima), impactos directos del clima sobre la salud (consecuencias directas del clima), e impactos mediados por instituciones humanas (directamente atribuibles

Tabla 2. Clasificación del impacto del cambio climático en la salud humana, IPCC 2014

\begin{tabular}{l}
\hline Impactos mediados por el ecosistema \\
Enfermedades transmitidas por vectores \\
- Malaria, dengue, garrapatas, otras \\
Infecciones transmitidas por agua y alimentos \\
- Vibrio, otros parásitos, bacterias, y viruses \\
Calidad de aire \\
- Exposiciones ozono a largo-plazo \\
- Episodios agudos de contaminación del aire \\
- Aeroalérgenos \\
Impactos directos del clima sobre la salud \\
Impactos relacionados al calor y al frio \\
Inundaciones y tormentas \\
Radiación ultravioleta \\
Impactos mediados por instituciones humanas \\
Nutrición \\
Salud ocupacional \\
- Golpe de calor y shock por calor \\
- Agotamiento por calor y pérdida de capacidad de trabajo \\
- Otras preocupaciones ocupacionales \\
\hline
\end{tabular}

Traducido y Resumido de: IPCC WGII AR5 Chapter 11. Human Health: Impacts, Adaptation, and Co-Benefits. 2014

Disponible en: https://www.ipcc.ch/pdf/assessment-report/ar5/wg2/drafts/ fd/WGIIAR5-Chap11_FGDall.pdf 
a la actividad humana). Si bien las instituciones humanas tienen mucho que ver con la presencia de enfermedad en cualquiera de estas categorías, se considera que aquellas en la última categoría son las más determinantes para los fines de las instituciones humanas (por ejemplo, salud ocupacional). También tienden a ser más crónicas y difíciles de medir (por ejemplo, nutrición y salud mental).

\section{INDICADORES}

Los indicadores son medidas cuantitativas de resumen que pueden ser utilizados para monitorear cambios en las condiciones por persona, lugar y tiempo. Los indicadores de salud ambiental son necesarios para medir la vulnerabilidad actual de las poblaciones al cambio de clima y para monitorear los posibles cambios en la índices de salud con el propósito de determinar si el cambio climático está afectando realmente su rango geográfico e incidencia ${ }^{(12)}$. Para el IPCC, la vulnerabilidad es una función de la exposición, la sensibilidad y la capacidad de adaptación, y se desarrollan indicadores para estas tres dimensiones.

Desde hace mucho existe una gran cantidad de debate acerca de la necesidad de desarrollar nuevas formas de evaluar y monitorear la susceptibilidad de las poblaciones a los factores ambientales que producen enfermedad ${ }^{20)}$. Gran parte del debate se centra en el requisito de que los indicadores sean relativamente fáciles de recoger y rápidos para aplicar. Sin embargo, en el afán de encontrar indicadores existentes, o para desarrollar otros nuevos, que sean útiles, se deben tener en cuenta otros atributos importantes del indicador, por ejemplo: 1) si el indicador representa verdaderamente una relación causal subyacente entre una exposición del medio ambiente y una consecuencia de la salud; o 2) si el indicador propuesto es una estimación razonablemente válida del factor causal subyacente. La mayoría de las asociaciones en epidemiología son solo estadísticas y, por lo tanto, no establecen causalidad. Si no hay una relación de causaefecto entre la enfermedad trazadora y el cambio climático, los cambios en la prevalencia de una enfermedad trazadora pueden no reflejar necesariamente el efecto del cambio climático sino, por ejemplo, podrían ser una medida de la eficiencia de los programas de salud pública (por ejemplo, vacunación o tratamiento).

Los indicadores no siempre incluyen solo los efectos en salud. Se considera que los indicadores pueden ser: de cambio climático, de salud ambiental, de vulnerabilidad o de impacto/efectos en la salud. Muchas de las propuestas para cuantificar los efectos en salud del cambio climático proponen la adopción de indicadores de la exposición a eventos y peligros del clima tales como el número de días de calor extremo, el número de inundaciones, y la prevalencia de los vectores de enfermedades infecciosas. En algunos casos se preferirá estudiar indicadores de vulnerabilidad (es decir, de riesgo), y se hará el monitoreo de los cambios en la vulnerabilidad (análisis de riesgo), no necesariamente de los efectos. En los Estados Unidos, la NCA anticipa que los indicadores del cambio climático serán seleccionados como parte de un proceso para aumentar la comprensión de los tipos de cambio y umbrales en apoyo de la toma de decisiones.

La Colaboración Estatal para Indicadores del Estado de Salud Ambiental (State Environmental Health Indicator Collaborative), un subcomité del Consejo de Epidemiólogos Estatales y Territoriales (Council of State and Territorial Epidemiologists o CSTE por sus siglas en inglés), describe los "indicadores de salud ambiental" como: los elementos ambientales de las fuentes, peligros, riesgos, efectos sobre la salud y actividades de prevención e intervención que pueden ser utilizados para evaluar determinantes ambientales positivos y negativos para la salud (21). Este subcomité de indicadores de cambio climático del CSTE seleccionó indicadores que medían la vulnerabilidad actual a la variabilidad del clima y el cambio o medidas de las variables ambientales que pueden afectar directa o indirectamente a la salud humana. Los indicadores también fueron seleccionados para realizar un seguimiento de los posibles cambios en los resultados de salud para determinar si el cambio climático está afectando realmente la incidencia en su área de distribución geográfica. Los criterios incluyen la temporalidad de datos, la integridad y la disponibilidad.

En el 2009, el subcomité de indicadores de cambio climático del CSTE publicó un reporte titulado "Indicadores de Salud Ambiental para Cambio Climático en los Estados Unidos". Este documento propone 27 indicadores en seis categorías: ambientales, morbilidad y mortalidad, vulnerabilidad, mitigación, adaptación, e indicadores de políticas política (Tabla 3). El subcomité del CSTE también desarrolló manuales de instrucción para completar cada indicador de cambio climático. La lista de indicadores del CSTE incluye las fuentes donde se pueden encontrar estos datos, los años para los cuales se pueden obtener información confiable, y las limitaciones que se conocen sobre los datos obtenidos ${ }^{(22)}$. Por ejemplo, datos sobre las temperaturas máximas y mínimas a nivel nacional se pueden obtener del Centro Nacional de Datos Climáticos (National Climatic Data Center o NCDC por sus siglas en inglés) donde se tienen registros desde el año 1900 a la fecha para los Estados Unidos. EI NCDC se considera el archivo de datos climáticos más grande del mundo. Sin embargo, el factor limitante es que las estaciones de monitoreo no están siempre en centros poblados. 
Tabla 3. Indicadores propuestos de salud ambiental para cambio climático, CSTE 2009

\begin{tabular}{|c|}
\hline Indicadores ambientales \\
\hline - Gases de efecto invernadero \\
\hline • Eventos de masas de aire "estancadas" \\
\hline - Ozono \\
\hline - Temperaturas máxima y mínima (índice de calor) \\
\hline - Alertas de olas de calor \\
\hline - Conteo de polen \\
\hline - Incendios forestales \\
\hline - Sequías \\
\hline - Brotes de algas peligrosas \\
\hline Indicadores de vulnerabilidad \\
\hline -Ancianos viviendo solos \\
\hline • Índice de pobreza \\
\hline - Riesgo de inundaciones \\
\hline - Elevación del nivel del mar \\
\hline Indicadores de mitigación \\
\hline - Eficiencia energética \\
\hline - Uso de energía renovable \\
\hline - Millas de vehículo recorridas \\
\hline Indicadores de adaptación \\
\hline -Acceso a centros de enfriamiento \\
\hline - Número de sistemas de alerta para olas de calor \\
\hline $\begin{array}{l}\text { - Número de planes municipales para el fenómeno de "Isla } \\
\text { urbana de calor" }\end{array}$ \\
\hline $\begin{array}{l}\text { - Número de sistemas de vigilancia en salud relacionados } \\
\text { al cambio climático }\end{array}$ \\
\hline $\begin{array}{l}\text { - Personal de salud asequible y entrenado en cambio } \\
\text { climático }\end{array}$ \\
\hline Indicadores de morbilidad y mortalidad \\
\hline - Mortalidad por calor extremo \\
\hline - Morbilidad por calor extremo \\
\hline - Lesiones/muertes por eventos extremos de clima \\
\hline - Casos humanos de infecciones ambientales \\
\hline - Enfermedad respiratoria por contaminación del aire \\
\hline - Enfermedad respiratoria alérgica por polen \\
\hline Indicadores de políticas \\
\hline - Ciudades incluidas den el Protocolo de Kyoto \\
\hline $\begin{array}{l}\text { - Ciudades participando en iniciativas sobre cambio } \\
\text { climático }\end{array}$ \\
\hline
\end{tabular}

Traducido de: Environ Health Perspect 117:1673-1681 (2009)
Por otro lado, si bien se considera que el NCDC puede producir una lista de todos los eventos extremos de clima desde el año 1900, las lesiones y muertes asociadas a dichos eventos se consideran subrreportadas y poco confiables. Otros indicadores, como los de adaptación y de políticas, aún tienen que ser creados y se tiene que identificar la institución responsable de monitorear esos datos. Finalmente, se puede apreciar de la lista del CSTE que para evaluar los efectos del cambio climático en la salud de las personas no se precisa de la participación exclusiva del sector Salud, sino que varios sectores aportan con sus insumos para establecer el análisis. Posteriormente, el CSTE evaluó el periodo necesario para completar cada indicador, la utilidad percibida para los indicadores propuestos, y la posibilidad de que estos indicadores sean adoptados por los departamentos de salud regionales.

De acuerdo con los usos propuestos para los indicadores, la medición de tendencias de resultados/ efectos asociados a la salud (por ejemplo, de mortalidad y morbilidad) puede centrarse en los peligros relacionados con el clima y no en medidas más amplias de la vulnerabilidad (por ejemplo, los indicadores de la exposición, la capacidad de adaptación). Por ejemplo, para un evento específico como una ola de calor, se puede definir la vulnerabilidad al cambio climático como una función de: 1) el nivel de exposición a un evento de ola de calor (temperatura y humedad); 2) el nivel de sensibilidad a un evento de ola de calor (acceso a aire acondicionado), y 3) el nivel de capacidad de adaptación de las poblaciones (medidas para refrescarse y mantenerse hidratado) ${ }^{(23)}$. Los indicadores también pueden ayudar a distinguir entre, por ejemplo, todas las muertes durante una ola de calor y aquellas muertes "atribuibles" al cambio climático.

Ante la propuesta de múltiples escenarios futuros posibles, se hace complicado desarrollar políticas en salud inteligentes para un futuro indeterminado debido a la incertidumbre al predecir correctamente el cambio en el ambiente y las decisiones humanas ${ }^{(26)}$. Sin embargo, se reconoce que la mayoría de los riesgos para la salud asociados al cambio climático ya existen y potencialmente ya podrían estar siendo monitorizados ${ }^{(24)}$.

\section{EL CASO PERUANO}

El Perú es una experiencia singular no solo por su diversidad ecológica, sino también por sus limitaciones. En un país con escasos datos meteorológicos históricos, los modelos matemáticos son inestables lo que hace poco confiables las predicciones sobre el clima. La tarea que tiene el Servicio Nacional de Metereología e Hidrología (SENAMHI) es monumental: desarrollar escenarios de cambio climático a nivel nacional para el año 2030. Sería 
bastante pedirle a una institución que haga predicciones sobre el clima (décadas hacia el futuro) con los pocos datos disponibles, pero es poco realista pedirle que haga tales predicciones cuando simplemente no hay datos. El Perú no tiene suficientes estaciones meteorológicas funcionando por suficiente tiempo para generalizar tendencias en todo el país. Cada modelo estadístico implica una generalización con un cierto margen de error, incluso aquellos hechos en Estados Unidos, con miles de estaciones de monitoreo y décadas de datos climatológicos. La técnica propuesta por el SENAMHI (el downscaling o reducción de escala) implica "aterrizar" en el Perú generalizaciones cada vez más lejos de los datos reales. Adicionalmente, existen tantos tipos de modelos climáticos que los reportes más recientes del IPCC establecen un "promedio" de todos los modelos para calificar sus proyecciones ("concordancia" alta o baja). Lamentablemente, en el Perú son pocas las publicaciones que analizan críticamente los efectos en la salud del cambio climático propuestos por publicaciones internacionales.

Efectivamente, la mayoría de la evidencia proviene de países en desarrollo y esa información es útil, siempre y cuando se adapte al contexto peruano. Por ejemplo, no podemos hablar de las causas del cambio climático en el Perú sin mencionar la deforestación. La deforestación y la degradación de los bosques no solo empeoran el problema actual al privarnos de la actividad "limpiadora" de la vegetación, sino que también contribuyen a las emisiones de gases de efecto invernadero mediante la combustión de la biomasa forestal y la descomposición del material vegetal restante y el carbono en el suelo que habían acumulado $\mathrm{CO}_{2}$ durante décadas ${ }^{(25)}$. Las "turberas" tropicales (el sustrato donde crecen nuestras selvas amazónicas) son una importante fuente de $\mathrm{CO}_{2}$ que se devuelve a la atmósfera al quemar los bosques tropicales. Esto es importante para el Perú, pero no se menciona en algunas revisiones peruanas que tratan sobre el tema. Tampoco podemos adoptar ciegamente la preocupación de países industrializados por la reemergencia de enfermedades infecciosas que son endémicas en Perú. Hace varios años que los modelos estadísticos se volvieron más complejos y le quitaron inercia al pánico inicial causado por las enfermedades infecciosas.

Otra preocupación extranjera es que se afecte el acceso a agua potable (no solo libre de patógenos sino también libre de contaminantes químicos) mientras que en el Perú actualmente no existe acceso a agua potable para muchas comunidades en la selva debido a los derrames de petróleo y en la sierra debido a la actividad minera. Con o sin cambio climático deberíamos estar resolviendo estos problemas de salud. Como ya se explicó, para la salud pública del Perú la adaptación tiene que ser más importante que la mitigación porque es algo que podemos controlar. Otro concepto confundido es la Oscilación Sur de EI Niño (EI Niño Southern Oscilation o ENSO por sus siglas en inglés) que como su nombre lo indica es un sistema cíclico que se repite todos los años.

El fenómeno El Niño es una aberración climática cuya intensidad actualmente no se puede predecir con suficiente anticipación. Si queremos prevenir los efectos de El Niño, tenemos los "datos", solo falta convertirlos en información ${ }^{(26)}$.

Finalmente, los peruanos debemos desconfiar de publicaciones que llaman a los "altos niveles de dirección en salud" y que proponen la creación de nuevas "instituciones específicas para cambio climático". No las vamos a mencionar aquí para no fomentar la desinformación, pero no están entendiendo realmente el problema. El "problema" del cambio climático tiene una explicación física y científica, pero las "consecuencias" en la salud de la población, y por tanto, las posibles "soluciones" a esas consecuencias, tienen matices sociales, económicos, y por lo tanto políticos. Esto quiere decir que en el Perú (bajo la legislación actual) el sector salud no puede resolver estos problemas (pobreza, vivienda, agua potable, nutrición, contaminación, etc.) que tienen implicancias esenciales en la salud de la población. Las soluciones deben venir de todos los sectores y de todos los niveles. Si entendemos de una vez que el cambio climático ya se está produciendo, para evaluar sus efectos solo tenemos que evaluar la situación actual de la salud de nuestra población. Si queremos que estén mejor preparados para enfrentar cambios en el clima, hay que mejorar su nivel de vida.

\section{CONCLUSIONES}

La salud de una población no es competencia exclusiva del sector Salud o del Ministerio de Salud. La historia nos demuestra que los cambios en el ambiente generados por otros sectores (como el acceso a agua potable, vivienda y electricidad) tienen una repercusión muy importante en la salud pública de cualquier población. Sin importar cual sea la clasificación de los impactos en la salud por el cambio climático, para poder prevenir o evitar muchos de los efectos potenciales en salud debemos: 1) identificar poblaciones susceptibles, vulnerables o desplazadas; 2) mejorar la infraestructura en salud publica, atención médica y vigilancia epidemiológica; 3) desarrollar capacidades y habilidades en modelamiento y predicción en salud, y 4) mejorar la comunicación del riesgo, la educación y la prevención en salud pública. Las intervenciones no tienen que ser inventadas, pueden ser mejoradas o adaptadas en cada región para prevenir morbilidad y mortalidad adicional asociada al cambio climático. De la misma manera, los sistemas actuales de vigilancia epidemiológica y monitoreo sanitario deben ser adaptados para ser eficientes en el contexto futuro con los indicadores que se van a priorizar para medir con precisión la morbilidad y mortalidad atribuible al cambio climático. Es decir, el cambio climático trae poco cambio para la salud pública del Perú: la lucha contra los efectos en salud del cambio climático es la lucha contra la pobreza y la desigualdad, y eso no es nada nuevo en la salud pública del Perú.

Fuentes de financiamiento: autofinanciado

Conflictos de interés: ninguno 


\section{REFERENCIAS BIBLIOGRÁFICAS}

1. Naciones Unidas. Convención Marco de las Naciones Unidas sobre Cambio Climático (CMNUCC) [internet]. Rio de Janeiro: Naciones Unidas; 1992 [citado el 15 de marzo de 2016]. Disponible en: http://unfccc.int/ resource/docs/convkp/convsp.pdf

2. IPCC Fourth Assessment Report: Climate Change 2007. Informe del Grupo de Trabajo I-Base de las Ciencias Físicas. RT.4 Comprensión y Atribución del Cambio Climático [internet]. Geneva: IPCC; 2007 [citado 15 de marzo de 2016]. Disponible en: https://www.ipcc.ch/ publications_and_data/ar4/wg1/es/ tssts-4.html

3. Parry ML, Canziani OF, Palutikof JP, van der Linden PJ, Hanson CE, editores. Climate Change 2007: Impacts, Adaptation and Vulnerability. Contribution of Working Group II to the Fourth Assessment Report of the Intergovernmental Panel on Climate Change 2007. Cambridge: IPCC; 2007.

4. Nobelprize.org [internet]. The Nobel Peace Prize; 2007. [actualizado el 15 de marzo de 2016; citado 15 de marzo de 2016]. Disponible en: http://www. nobelprize.org/nobel_prizes/peace/ laureates/2007/

5. climate.nasa.gov [internet].California: National Oceanic and Atmospheric Administration [actualizado el 14 de marzo de 2016; citado 15 de marzo de 2016] Disponible en: http://climate. nasa.gov/evidence/

6. Fred Pearce. Countdown to Chaos. Chicago: New Scientist; 1997 [citado 15 de marzo de 2016]. Disponible. https://www.newscientist.com/ article/mg15621065-100-countdownto-chaos-time-signals-keep-the-worldin-step-tweak-them-just-a-little-andlife-as-we-know-it-will-quickly-grindto-a-halt-says-david-kestenbaum/

7. Intergovernmental Panel on Climate Change. Working Group II. Fifth Assessment Report. Chapter 11. Human Health: Impacts, Adaptation, and Co-Benefits [internet]. Ginebra: IPCC; 2014 [citado 15 de marzo de 2016]. Disponible en: https://www. ipcc.ch/pdf/assessment-report/ar5/
wg2/drafts/fd/WGIIAR5-Chap11_ FGDall.pdf

8. National Oceanic and Atmospheric Administration (NASA) [internet]. California: Global Climate Change. Graphic: The relentless rise of carbon dioxide; 2016 [actualizado el 14 de marzo de 2016; citado 15 de marzo de 2016]. Disponible en: http://climate. nasa.gov/climate_resources/24/

9. Hadley Centre for Climate Prediction and Research. Climate Change and its Impacts. Stabilisation of $\mathrm{CO} 2$ in the atmosphere [internet]. London: Hadley Centre for Climate Prediction and Research; 1999 [citado 15 de marzo de 2016]. Disponible en: http://www.metoffice.gov.uk/media/ pdf/q/9/COP5.pdf

10. ncdc.noaa.gov [internet]. Asheville, NC: National Oceanic and Atmospheric Administration (NOAA). National Centers for Environmental Information. Global Climate Change Indicators; 2016; [actualizado el 17 de marzo de 2016; citado 15 de marzo de 2016]. Disponible en: https://www.ncdc. noaa.gov/indicators/

11. Intergovernmental Panel on Climate Change (IPCC) - Working Group II: Impacts, Adaptation and Vulnerability. Third Assessment Report. 11.2.4.6. Potential Impacts and Coastal Zone Management. Disponible en: http:// www.ipcc.ch/ipccreports/tar/wg2/ index.php?idp $=446$

12. Intergovernmental Panel on Climate Change (IPCC) - Working Group II: Impacts, Adaptation and Vulnerability. Fifth Assessment Report. Chapter 5. 2014. Disponible en: https://ipccwg2.gov/AR5/report/full-report/

13. National Oceanic and Atmospheric Administration. (NOAA) study: Human-caused climate change a major factor in more frequent Mediterranean droughts. Octubre 2011. Washinton D.C.: NOAA; 2011 [Citado el 15 de marzo de 216]. Disponible en: http://www.noaanews.noaa.gov/ stories2011/20111027_drought.html

14. Naciones Unidas. Protocolo de Kyoto de la Convención Marco de las Naciones Unidas sobre el Cambio Climático [internet]. Kyoto: Naciones Unidas; 1998 [citado el 15 de marzo de 2016]. Disponible en: http://unfccc. int/kyoto_protocol/items/2830.php

15. Dhainaut JF, Claessens YE, Ginsburg C, Riou B. Unprecedented heat-related deaths during the 2003 heat wave in Paris: consequences on emergency departments. Crit Care. 2004;8(1):1-2.

16. Semenza JC1, McCullough JE, Flanders WD, McGeehin MA, Lumpkin JR. Excess hospital admissions during the July 1995 heat wave in Chicago. Am J Prev Med. 1999;16(4):269-77.

17. Organización Mundial de la Salud. Centro de Prensa. Cambio climático y salud. Septiembre 2015. Nota descriptiva $\mathrm{N}^{\circ}$ 266. Ginebra: OMS; 2015 [Citado el 13 de febrero de 216] Disponible en http://www.who.int/ mediacentre/factsheets/fs266/es/.

18. The World Bank. Turn down the heat. Why a $4^{\circ} \mathrm{C}$ warmer world must be avoided. A report for the World Bank by the Potsdam Institute for Climate Impact Research. Washington, DC: The World Bank, 2012.

19. National Institute of Health (NIH) National Institute of Environmental Health Sciences (NIEHS). The Interagency Working Group on Climate Change and Health. A Human Health Perspective on climate change. April 22, 2010)

20. Pastides H. An epidemiological perspective on environmental health indicators. World Health Stat Q. 1995;48(2):140-3.

21. Climate Change Indicators Subcommittee of the Council of State and Territorial Epidemiologists (CSTE). Environmental Health Indicators for Climate Change. Discussion Paper for National Climate Assessment. Atlanta: Climate Change Indicators Subcommittee of the Council of State and Territorial Epidemiologists (CSTE); 2012 [citado 15 de marzo de 2016]. Disponible en: http://c.ymcdn. com/sites/www.cste.org/resource/ resmgr/environmentalhealth / ncarecommendation22312.pdf 
22. English PB, Sinclair AH, Ross $\mathrm{Z}$, Anderson $\mathrm{H}$, Boothe V, Davis $\mathrm{C}$, et al. Environmental Health Indicators of Climate Change for the United States: findings from the State Environmental Health Indicator Collaborative. Environ Health Perspect. 2009;117(11):1673-81. doi: 10.1289/ehp.0900708.

23. Zhu Q, Liu T, Lin H, Xiao J, Luo Y, Zeng W, et al. The spatial distribution of health vulnerability to heat waves in Guangdong Province, China. Glob Health Action. 2014;7:25051. doi: 10.3402/gha.v7.25051.
24. Ebi KL. Public health responses to the risks of climate variability and change in the United States. J Occup Environ Med. 2009;51(1):4-12. doi: 10.1097/ JOM.0b013e31816fd67b.

25. van der Werf GR, Morton DC, DeFries RS, Olivier JGJ, Kasibhatla PS, Jackson $\mathrm{RB}$, et al. CO2 emissions from forest loss. Nature Geoscience. 2009;2:73738. doi:10.1038/ngeo671

26. Suarez-Ognio L, Estela-Ayamamani D, Cáceres-Mejía B, Gambirazio-Carbajal C, Cabrera R. Impacto del Fenómeno "El Niño" de 1997-1998 en la Salud de la Población Peruana, Riesgo Potencial para el 2015. Rev Peru Med Exp Salud Publica. 2015;32(2):403-4. doi:10.17843/ rpmesp.2015.322.1645

\section{https://www.youtube.com/RPMESP}

Correspondencia: Carlos A. Sánchez Zavaleta Dirección: Av. Horacio Urteaga 2080 dpto. 305. Lima, Perú

Telefono: (511) 995756027

Correo electrónico: csanchez.peru@gmail.com

MEDICINA EXPERIMENTAL

Y SALUD PÚBLICA
Difundiondo investigación en salud ta

RPMESP

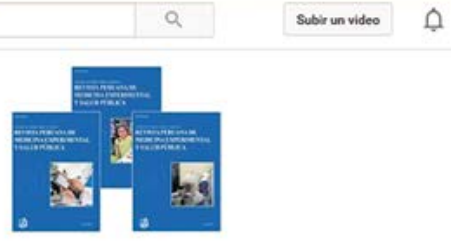

La Revista Peruana de Medicina Experimental y Salud Publica es el organo oficial de difusión cientifica del Instituto Nacional de Salud. Mostrar más

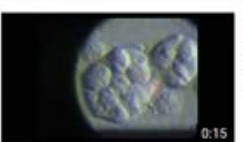

Trofozoito de Trichomonas hominis con siete Blastocystis fagocitados

Hace 1 dia. -6 vistas

se observa un trofozolito de Trichomonas hominis con siete Blastocystis fagocitados

(izquierda) y una Trichomona mas pequeña con tres Blastocystis fagocitados (derechlo)

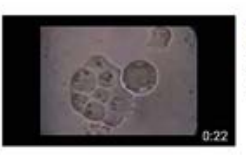

Trofozoito de Trichomonas hominis con siete Blastocystis fagocitados

Hace 1 dia. -4 vistas

Se obsema un wollozolto de Trichomonas hominis con siete Blastocystis fagocitados

intento de fagccitosis de Blastocystis por un cilostoma elistico de Trichomonas.

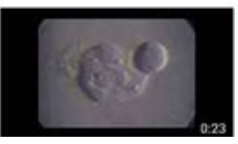

Trofozoito de Trichomonas hominis con ocho Blastocystis fagocitados

Hace 1 dia. -8 vistas

.

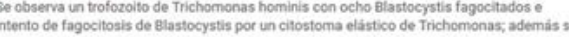

